\title{
Population dynamics of plateau pika under lethal control and contraception control
}

\author{
Hanwu Liu ${ }^{1,2,3^{*}}$, Zhen $\mathrm{Jin}^{2}$, Yuming Chen ${ }^{3,4}$ and Fengqin Zhang ${ }^{3}$
}

\author{
* Correspondence: liuhanwu- \\ china@163.com \\ 'Electromechanical Engineering \\ College, North University of China, \\ Taiyuan 030051, China \\ Full list of author information is \\ available at the end of the article
}

\begin{abstract}
The overabundance of plateau pika brings a great damage to the alpine meadow. Rodenticide and sterilant have been used to control this mammalian pest. In this article, we proposed models to incorporate these controls and the seasonal cycle of breeding and non-breeding. It is shown that when the basic reproduction number is less than 1 then the trivial equilibrium is globally asymptotically stable; if the basic reproduction number is greater than 1 then the trivial equilibrium is unstable and there is a positive equilibrium which attracts all positive solutions. Then we study the effects of controls on the existence of the positive equilibrium and the population size. These theoretical results are supported by numerical simulations. We also propose the possible strategies to be implemented in practice.

Mathematical Subject Classification: 39A30; 39A60; 92D25.
\end{abstract}

Keywords: contraception control, lethal control, seasonal breeding, mathematical model, plateau pika

\section{Introduction}

The plateau pika (Ochotona curzoniae) is a keystone species of the Qinghai-Tibet plateau. Its presence of reasonable size is important for the healthy development and the biodiversity of the alpine meadow. In the recent years, due to overgrazing and the global climate change, the alpine meadow has degraded seriously. The degraded meadow provides the plateau pika with a better habitat and results in the overabundance of plateau pika. Now, plateau pika is regarded as a pest because its competition with livestock for herbage and its burrowing activity which leads to soil erosion and vegetation disturbances [1-4].

In order to manage rodent pests, a variety of methods have been used [5]. The commonly used one to control plateau pika is poisoning, which kills them with botulins of models $C$ and D [6]. Now, sterilants $[7,8]$ have also been used to control plateau pika $[9,10]$. Zhang [11] argued that culling and contraception combined will give better result in controlling population numbers. In fact, some sterilants can also poison target species [10].

Reproduction in mammals is likely affected by the resources available in its habitat to sustain the energy demands of reproduction. Plateau pika is a native herbivorous species inhabiting the Qinghai-Tibetan plateau. They have short spring-summer breeding seasons typical to herbivorous small mammals inhabiting alpine environments [12]. Considered the seasonal reproduction of plateau pika, we proposed several discrete

(c) 2012 Liu et al; licensee Springer. This is an Open Access article distributed under the terms of the Creative Commons Attribution License (http://creativecommons.org/licenses/by/2.0), which permits unrestricted use, distribution, and reproduction in any medium, provided the original work is properly cited. 
models to understand the dynamics of plateau pika. During the breeding and the nonbreeding seasons, the dynamics of plateau pika population satisfies different models. This kind of model can describe the population dynamics in more detail. With this kind of model, Burkey and Stenseth [13] studied the effects of resource patchiness and the effects of a seasonally variable environment on the population dynamics of a herbivore species, and Liu et al. [14] investigated the influence of environment change on population dynamics.

This article is structured as follows. In Section 2, we model the population with natural growth. We take into account the seasonal cycle of breeding and non-breeding. Then, in Section 3, we modify the model obtained in Section 2 to incorporate lethal control and contraception control. It is shown that when the basic reproduction number is less than 1 then the trivial equilibrium is globally asymptotically stable; if the basic reproduction number is greater than 1 then the trivial equilibrium is unstable and there is a positive equilibrium which attracts all positive solutions. In Section 3, we also study the effects of controls on the existence of positive equilibrium and the population size. To support the theoretical results, numerical simulations are presented in Section 4, and finally the article is concluded with a short discussion.

\section{A model for natural growth}

Assume that the breeding season of plateau pika is from April to August and the nonbreeding season is from September to the next March. Let $x_{n}$ and $y_{n}$ denote the population size of the plateau pika in April and August of the nth year, respectively. During the non-breeding season, the population size decreases gradually due to death. The ratio of the population size in April to that in the previous August is called the overwintering survival rate. During the breeding season, some newborns are recruited and some individuals will die. The ratio of the population size in August to that in April is called the increasing rate. Note that the number of plateau pika in August is the sum of the amount of newborns and the amount of survived individuals during the breeding season. Indeed, the birth rate refers to the ratio of amount of newborns in August to the population size in April and the survival rate during the breeding season refers to the ratio of amount of survived overwintering individuals in August to the population size in April.

We assume that both overwintering survival rate and increasing rate are density dependent and they decrease as the population densities increase. More precisely, we assume that the overwintering survival rate is $f(x)=a /(b+x)$ and the increasing rate is $g(x)=c /(d+x)$, where $a, b, c$, and $d$ are all positive. From the meanings of $f$ and $g$, we have $\max \{f(x)\}<1$ and $\max \{g(x)\}>1$, which imply $0<a<b$ and $0<d<c$. In the sequel, we always assume that $0<a<b$ and $0<d<c$. We can also rewrite $g(x)$ as $g(x)=B(x)+D(x)$, where $B(x)=c(1-\varepsilon) /(d+x)$ and $D(x)=c \varepsilon /(d+x)$ are the birth rate and death rate during the breeding season, respectively. Again, from $\max \{D(x)\}<1$, we get $0<\varepsilon<d / c$.

From the above discussion, we can propose the following model for natural growth,

$$
\left\{\begin{array}{l}
y_{n}=\frac{c x_{n}}{d+x_{n}}, \\
x_{n+1}=\frac{a y_{n}}{b+y_{n}},
\end{array}\right.
$$


or equivalently

$$
x_{n+1}=\frac{a c x_{n}}{b d+(b+c) x_{n}} \triangleq H\left(x_{n}\right) .
$$

From the view of biology, we assume that $x_{0} \geq 0$. It is easy to see that $x_{n} \geq 0$ when $x_{0} \geq 0, n \in \mathbb{N} \triangleq\{0,1, \ldots\}$. Moreover, if $x_{0}>0$ then $x_{n}>0$ for all $n \in \mathbb{N}$. In fact, the solution of (1) can be found by mathematical induction. If $a c \neq b d$, then

$$
x_{n}=\frac{(a c)^{n}(a c-b d) x_{0}}{(b d)^{n}(a c-b d)+(b+c)\left[(a c)^{n}-(b d)^{n}\right] x_{0}} ;
$$

while if $a c=b d$,

$$
x_{n}=\frac{a c x_{0}}{a c+n(b+c) x_{0}} .
$$

Obviously, $x^{*}=0$ is always an equilibrium of (1), denoted by $O_{1}$. If $a c>b d$, then (1) has another unique positive equilibrium $x^{*}=(a c-b d) /(b+c)$, denoted by $E_{1}$. The following result follows directly from (2) and (3).

\section{Theorem 1.}

(i) If $a c \leq b d$, then $O_{1}$ is globally asymptotically stable.

(ii) If $a c>b d$, then $O_{1}$ is unstable but $E_{1}$ asymptotically attracts all positive solutions.

When $x=0, f(x)$ takes the maximum value $a / b$, which is called the intrinsic overwintering survival rate. Similarly, when $x=0, g(x)$ takes the maximum value $c / d$, which is called the intrinsic increasing rate. As a result, we call $(a / b)(c / d)=a c / b d$ the basic reproduction number and is denoted by $R_{0}$. Then, Theorem 1 tells us that if $R_{0} \leq 1$ then the population dies out; otherwise, (1) possesses a globally asymptotically stable positive equilibrium and hence the population exists persistently.

Moreover, at $E_{1}$, the annual increasing rate equals one and the population size at August is $y^{*}=(a c-b d) /(a+d)$. When the increasing rate during the breeding season equals one, that is $f(x)=1$, the population size at April is $x^{\#}=c-d$, which may be called the carrying capacity. Clearly, $x^{\#}>y^{*}>x^{*}$. In other words, the population at the annual positive equilibrium is smaller than that at the positive equilibrium of the breeding season.

\section{A model for the controlled population}

\subsection{Model formulation}

Recall that when $a c \leq b d$, the natural plateau pika population dies out gradually and hence there is no need to control. But, when $a c>b d$, the population exists persistently and is harmful after reaching high density. So, control should be implemented. In the sequel, we always assume that $a c>b d$.

We assume that both the lethal and contraception controls are applied simultaneously at April of every $h$ years. Let $p$ and $q$ denote the contraception rate and removal (or killing) rate, respectively, i.e., $p \in(0,1)$ is the percentage of fertile individuals become sterilized instantaneously and $q \in(0,1)$ is the percentage of individuals instantaneously killed. Under control, the population is divided into fertile and sterile 
sub-populations. Let $f_{n}$ and $s_{n}$ denote the densities of fertile and sterile individuals in April at the $n$th year, respectively; $F_{n}$ and $S_{n}$ denote the densities of fertile and infertile individuals in August at the $n$th year, respectively.

Based on (1), if control is implemented at the $n$th year then we have

$$
\left\{\begin{aligned}
F_{n} & =\frac{c(1-q)(1-p) f_{n}}{d+(1-q)\left(f_{n}+s_{n}\right)}, \\
S_{n} & =\frac{c \varepsilon(1-q)\left(s_{n}+p f_{n}\right)}{d+(1-q)\left(f_{n}+s_{n}\right)} \\
f_{n+1} & =\frac{a F_{n}}{b+F_{n}+s_{n}}, \\
s_{n+1} & =\frac{a S_{n}}{b+F_{n}+S_{n}},
\end{aligned}\right.
$$

or equivalently,

$$
\left\{\begin{array}{l}
f_{n+1}=\frac{a c(1-q)(1-p) f_{n}}{b d+(1-q)(b+c-c p+c \varepsilon p) f_{n}+(1-q)(b+c \varepsilon) s_{n}} \\
s_{n+1}=\frac{a c \varepsilon(1-q)\left(s_{n}+p f_{n}\right)}{b d+(1-q)(b+c-c p+c \varepsilon p) f_{n}+(1-q)(b+c \varepsilon) s_{n}}
\end{array}\right.
$$

if there was no control implemented at the nth year then we have

$$
\left\{\begin{array}{c}
F_{n}=\frac{c f_{n}}{d+f_{n}+s_{n}}, \\
S_{n}=\frac{c \varepsilon s_{n}}{d+f_{n}+s_{n}} \\
f_{n+1}=\frac{a F_{n}}{b+F_{n}+S_{n}} \\
s_{n+1}=\frac{a S_{n}}{b+F_{n}+S_{n}}
\end{array}\right.
$$

or equivalently,

$$
\left\{\begin{array}{l}
f_{n+1}=\frac{a c f_{n}}{b d+(b+c) f_{n}+(b+c \varepsilon) s_{n}} \\
s_{n+1}=\frac{a c \varepsilon s_{n}}{b d+(b+c) f_{n}+(b+c \varepsilon) s_{n}}
\end{array}\right.
$$

Suppose that the control is implemented in April at the $n h$ th year $(n \in \mathbb{N}, h \geq 1)$. We use $\bar{f}_{n}, \bar{s}_{n}, \bar{F}_{n}, \bar{S}_{n}$ for $f_{n h}, s_{n h}, F_{n h}, S_{n h}$ respectively. Then it follows from (4) and (5) that

$$
\left\{\begin{array}{l}
\bar{f}_{n+1}=\frac{A \bar{f}_{n}}{B+C \bar{f}_{n}+D \bar{s}_{n}}, \\
\bar{s}_{n+1}=\frac{E\left(\bar{s}_{n}+p \bar{f}_{n}\right)}{B+C \bar{f}_{n}+D \bar{s}_{n}},
\end{array}\right.
$$


where

$$
\begin{aligned}
& A=(1-q)(1-p)(a c)^{h}, \\
& B=(b d)^{h} \\
& C=\frac{(1-q)(1-p)(b+c)}{b d-a c}\left[(b d)^{h}-(a c)^{h}\right]+\frac{p(1-q)(b+c \varepsilon)}{b d-a c \varepsilon}\left[(b d)^{h}-(a c \varepsilon)^{h}\right], \\
& D=\frac{(1-q)(b+c \varepsilon)}{b d-a c \varepsilon}\left[(b d)^{h}-(a c \varepsilon)^{h}\right], \\
& E=(1-q)(a c \varepsilon)^{h} .
\end{aligned}
$$

Note that $E<B$ as $a c \varepsilon<a c \cdot \frac{d}{c}=a d<b d$.

\subsection{Model analysis}

As before, we only need to consider solutions with non-negative initial conditions. Obviously, $O_{2}=(0,0)$ is always an equilibrium of $(6)$. Let $R_{2}=(1-q)(1-p)(a c)^{h}$ $(b d)^{h}$ and $Q_{2}=1-p-\varepsilon^{h}$. Then when $R_{2}>0$ (at that time $Q_{2}>0$ ) (6) has another unique positive equilibrium $E_{2}=\left(\bar{f}^{*}, \frac{\varepsilon^{h} p \bar{f}^{*}}{1-p-\varepsilon^{h}}\right)$, where $\bar{f}^{*}=\frac{(A-B)(A-E)}{A C-E C+p D E}$.

Note that, for a solution $\left(\bar{f}_{n}, \bar{s}_{n}\right)$ of (6), if $\bar{f}_{n_{0}}=0$ for some $n_{0} \in \mathbb{N}$ then $\bar{f}_{n}=0$ for $n \in$ $\mathbb{N}$ and if $\bar{f}_{0} \neq 0$ then $\bar{f}_{n} \neq 0$ for $n \in \mathbb{N}$. First, for a solution of (6) with $\bar{f}_{0} \neq 0$, dividing the second equation of (6) by the first one yields

$$
\frac{\bar{s}_{n+1}}{\bar{f}_{n+1}}=\frac{E}{A} \cdot \bar{s}_{n} \overline{\bar{f}}_{n}+\frac{p E}{A} \text {. }
$$

The above equation can be solved inductively to obtain

$$
\frac{\bar{s}_{n}}{\bar{f}_{n}}=\frac{\bar{s}_{0}}{\bar{f}_{0}}+n p
$$

if $Q_{2}=0$ (equivalently $A=E$ ) or

$$
\frac{\bar{s}_{n}}{\overline{\bar{f}}_{n}}=\left(\frac{\bar{s}_{0}}{\bar{f}_{0}}-\frac{p E}{A-E}\right)\left(\frac{E}{A}\right)^{n}+\frac{p E}{A-E}
$$

if $Q_{2} \neq 0$. Then, the first equation of (6) combined with (7) or (8) gives

$$
\bar{f}_{n}=\frac{\bar{f}_{0}}{\left[1+\frac{D \bar{s}_{0}+C \bar{f}_{0}}{B-A}+\frac{p A D \bar{f}_{0}}{(B-A)^{2}}\right]\left(\frac{B}{A}\right)^{n}+\frac{n p D \bar{f}_{0}}{A-B}+\frac{D \bar{s}_{0}+C \bar{f}_{0}}{A-B}-\frac{p A D \bar{f}_{0}}{(B-A)^{2}}}
$$

if $Q_{2}=0$ (at this time we have $A=E<B$ ) or

$$
\bar{f}_{n}=\frac{\bar{f}_{0}}{\frac{D}{E-A}\left(\bar{s}_{0}-\frac{p E \bar{f}_{0}}{A-E}\right)\left(\frac{E}{A}\right)^{n} \frac{n(A C-E C+P D E) \bar{f}_{0}}{A(A-E)}+1+\frac{D}{A-E}\left(\bar{s}_{0}-\frac{p E \bar{f}_{0}}{A-E}\right)}
$$

if $R_{2}=0$ (at this time we have $A=B>E$ and hence $Q_{2}>0$ ) or

$$
\bar{f}_{n}=\frac{\bar{f}_{0}}{\left[1+\frac{D \bar{S}_{0}}{B-E}+\frac{(E C-B C+p D E) \bar{f}_{0}}{(E-B)(B-A)}\right]\left(\frac{B}{A}\right)^{n}-\left[\frac{D \bar{s}_{0}}{B-E}-\frac{p D E \bar{f}_{0}}{(B-E)(A-E)}\right]\left(\frac{E}{A}\right)^{n}+\frac{(A C-E C+p D E) \bar{f}_{0}}{(A-B)(A-E)}}
$$


if $Q_{2} \neq 0$ and $R_{2} \neq 0$. With the help of (9)-(10), we can obtain the following result.

\section{Theorem 2 .}

(i) If $R_{2} \leq 0$ then $\mathrm{O}_{2}$ is asymptotically stable.

(ii) If $R_{2}>0$ then every solution with $\bar{f}_{0}=0$ tends to $\mathrm{O}_{2}$ but every solution with $\bar{f}_{0} \neq 0$ tends to $E_{2}$.

Proof. We only prove the case where $R_{2}>0$ since the proofs for the other cases are similar. In this case, we have $A>B>E$. First, assume that $\bar{f}_{0}=0$. Then $\bar{f}_{n}=0$ for $n \in$ $\mathbb{N}$ and hence

$$
\bar{s}_{n+1}=\frac{E \bar{s}_{n}}{B+D \bar{s}_{n}}
$$

by (6). It follows that

$$
\bar{s}_{n}=\frac{\bar{s}_{0}}{\left(1+\frac{D \bar{s}_{0}}{B-E}\right)\left(\frac{B}{E}\right)^{n}-\frac{D \bar{s}_{0}}{B-E}} \rightarrow 0 \quad \text { as } n \rightarrow \infty .
$$

Now, assume that $\bar{f}_{0} \neq 0$. Then $\bar{f}_{n}$ is given by (11) and hence $\bar{f}_{n} \rightarrow \frac{(A-C)(A-E)}{A C-E C+p D E}=\bar{f}^{*}$ as $n \rightarrow \infty$. This, combined with the first equation of (6) implies that $\lim _{n \rightarrow \infty} \bar{s}_{n}$ exists, say $\bar{s}$. Taking limits of both sides of the two equations in (6) yields,

$$
\left\{\begin{aligned}
\bar{f}^{*} & =\frac{A \bar{f}^{*}}{B+C \bar{f}^{*}+D \bar{s}^{\prime}} \\
\bar{s} & =\frac{E\left(\bar{s}+p \bar{f}^{*}\right)}{B+C \bar{f}^{*}+D \bar{s}} .
\end{aligned}\right.
$$

This gives $\bar{s}=\frac{\varepsilon^{h} p \bar{f}^{*}}{1-p-\varepsilon^{h}}$. In summary, we have proved $\left(\bar{f}_{n}, \bar{s}_{n}\right) \rightarrow E_{2}$ as $n \rightarrow \infty$ and hence the proof is complete.

Note that the intrinsic increasing rate of the fertile individuals is $\frac{A}{B}=\frac{(1-q)(1-p)(a c)^{h}}{(b d)^{h}}$, which is called the basic reproduction number with control and is denoted by $\tilde{R}_{0}$. It is easy to see that $\tilde{R}_{0}>1$ (respectively, $=1,<1$ ) is equivalent to $R_{2}>0$ (respectively, $=0,<0$ ). Then Theorem 2 can be rephrased as follows. If $\tilde{R}_{0} \leq 1$ then $O_{2}$ is globally asymptotically stable while if $\tilde{R}_{0}>1$ then $O_{2}$ is unstable and $E_{2}$ attracts all positive solutions.

\subsection{Parameter analysis}

In this section, based on (6), we study the effects of control strategies on the existence of $E_{2}$ and the (total) population size in April at $E_{2}$.

Recall that $E_{2}$ exists when $R_{2}>0$ or equivalently $(1-q)(1-p)>(b d / a c)^{h}$. This reveals the fact that the contraception rate $p$ and the removal rate $q$ have the same 
effect on determining whether the plateau pika population dies out. Moreover, for the existence of $E_{2}$, larger control period $h$ requires larger $p$ and/or $q$.

Now, under the assumption of the existence of $E_{2}$, we study the effects of control parameters on the population size in April at $E_{2}$, which is

$$
\Pi=\frac{\left(1-\varepsilon^{h}\right)\left[(1-q)(1-p)(a c)^{h}-(b d)^{h}\right]}{(1-q)(1-p) \Gamma+(1-q) \Lambda},
$$

where

$$
\begin{gathered}
\Gamma=\frac{(b+c)\left[(b d)^{h}-(a c)^{h}\right]}{b d-a c}-\frac{(b+c \varepsilon)\left[(b d)^{h}-(a c \varepsilon)^{h}\right]}{b d-a c \varepsilon}, \\
\Lambda=\frac{(b+c \varepsilon)\left[(b d)^{h}-(a c \varepsilon)^{h}\right]}{b d-a c \varepsilon}-\frac{\varepsilon^{h}(b+c)\left[(b d)^{h}-(a c)^{h}\right]}{b d-a c} .
\end{gathered}
$$

Clearly, the lethal and contraception controls have different roles in determining $\Pi$. Other effects are summarized below.

\subsubsection{Contraception control is better than the lethal control}

In particular, if $p=0$ (i.e., only lethal control is implemented) then the population size at $E_{2}$ is

$$
\Pi_{L}=\frac{\left[(a c)^{h}(1-q)-(b d)^{h}\right](b d-a c)}{\left[(b d)^{h}-(a c)^{h}\right](b+c)(1-q)}
$$

while if $q=0$ (i.e., only contraception control is implemented) then the population size at $E_{2}$ is

$$
\Pi_{S}=\frac{\left(1-\varepsilon^{h}\right)\left[(a c)^{h}(1-p)-(b d)^{h}\right]}{\frac{p(b+c \varepsilon)}{b d-a c \varepsilon}\left[(b d)^{h}-(a c \varepsilon)^{h}\right]+\frac{\left(1-p-\varepsilon^{h}\right)(b+c)}{b d-a c}\left[(b d)^{h}-(a c)^{h}\right]} .
$$

Thus, if $p=q$ then

$$
\frac{\Pi_{S}}{\Pi_{L}}=\frac{1-p-\varepsilon^{h}+p \varepsilon^{h}}{1-p-\varepsilon^{h}+\frac{p(b+c \varepsilon)(a c-b d)\left[(b d)^{h}-(a c \varepsilon)^{h}\right]}{(b d-a c \varepsilon)(b+c)\left[(a c)^{h}-(b d)^{h}\right]}} .
$$

This, combined with the fact that $\frac{(b+c \varepsilon)(a c-b d)\left[(b d)^{h}-(a c \varepsilon)^{h}\right]}{(b d-a c \varepsilon)(b+c)\left[(a c)^{h}-(b d)^{h}\right]}>\varepsilon^{h}$, implies $\Pi_{S}<\Pi_{L}$. Therefore, with only one control being implemented with the same rate, the contraception control is better than the lethal control.

\subsubsection{Increasing $p$ or $q$ will decrease $\Pi$}

Note that

$$
\frac{\partial \Pi}{\partial p}=\frac{\Gamma\left(1-\varepsilon^{h}\right)(1-q)\left[(1-q)(a c)^{h}-(b d)^{h}\right]}{-[(1-q)(1-p) \Gamma+(1-q) \Lambda]^{2}}<0 .
$$


Similarly, we have $\frac{\partial \Pi}{\partial p}<0$. It follows that increasing efficiency of either contraception control or lethal control will decrease the population size, which agrees with our intuition. Further increasing the rates will results the extinction of the population. Note that $\Pi(p, q)<\Pi(0,0)=\frac{a c-b d}{b+c}$ This implies that with control being implemented, the population size is always less than that in the natural growth.

\subsubsection{Increasing the control period $h$ will increase $\boldsymbol{I}$}

The derivative of $\Pi$ with respect to $h$ is

$$
\frac{\partial \Pi}{\partial h}=\frac{\frac{(1-\varepsilon)(a+d) b c p \varepsilon^{h} \ln \varepsilon}{(b d-a c \varepsilon)(b d-a c)\left(1-\varepsilon^{h}\right)^{2}}\left[z\left(\varepsilon^{h}\right)-z\left((b d / a c)^{h}\right)\right]\left(\frac{b d}{a c}\right)^{h} \ln \frac{b d}{a c}}{(1-q)\left\{\frac{p(b+c \varepsilon)}{b d-a c \varepsilon}+\left[\frac{b+c}{b d-a c}-\frac{b c p(1-\varepsilon)(a+d)}{(b d-a c \varepsilon)(b d-a c)\left(1-\varepsilon^{h}\right)}\right]\left[\left(\frac{b d}{a c}\right)^{h}-1\right]\right\}^{2}},
$$

where $z(x)=\frac{h(1-x)[(1-p)(1-q)-x]}{x \ln x}$. When $R_{2}>0$, we have $0<\varepsilon^{\mathrm{h}}<(b d / a c)^{\mathrm{h}}$ $<(1-p)(1-q)$. For $0<x<(1-p)(1-q), z(x)$ is an increasing function of $x$ since

$$
z^{\prime}(x)=\frac{h(1-x+\ln x)[x-(1-p)(1-q)]+x(x-1) \ln x}{(x \ln x)^{2}}>0 .
$$

Therefore, $\partial \Pi / \partial h>0$. This means that increase the control period will increase the population size. This again agrees with our expectation. Note that, as $h \rightarrow \infty, \Pi \rightarrow \frac{a c-b d}{b+c}$, the population size with no control.

\subsubsection{Increasing $\varepsilon$ will decrease II}

For this purpose, for $n \in \mathbb{N}$, we define a function $\phi_{n}:(0, \infty) \ni x \rightarrow 1+x+\ldots x^{n}$. Then one can easily show that for $a>1$ and $n \geq 1, \phi_{n}(a x) / \phi_{n}(x)$ is an increasing function of $x$. Note that we can rewrite $\Pi$ as

$$
\Pi=\frac{(1-p)(1-q)(a c)^{h}-(b d)^{h}}{(1-q)\left\{\frac{b+c}{a c-b d}\left[(1-p)(a c)^{h}-(b d)^{h}\right]+\frac{p b c(a+d)(b d)^{h-1} \varphi_{h-1}(a c \varepsilon / b d)}{(a c-b d) \varphi_{h-1}(\varepsilon)}\right\}} .
$$

Since $a c>b d$, one conclude that if $h>1$ then $\Pi$ is a decreasing function of $\varepsilon$ and if $h=1$ then $\Pi=\frac{(1-p)(1-q) a c-b d}{(1-q)(b+c-p c)}$ and hence $\varepsilon$ has no effect on $\Pi$.

Note that the parameters $p, h$, and $\varepsilon$ affect not only the population size at $E_{2}$, but also the ratio of fertile individuals to sterile individuals at $E_{2}$. This can easily be seen from $\frac{\bar{s}^{*}}{\bar{f}^{*}}=\frac{\varepsilon^{h} p}{1-p-\varepsilon^{h}}$

\section{Numerical simulations}

From April 2007 to September 2008, Liu et al. [15] executed field contraception experiment of plateau pika at Dawu Town, Maqin County of Qinghai Province. They selected 16 sample plots of 100 ha which are more than $0.5 \mathrm{~km}$ apart. Four replications of four treatments (quinestrol, levonorgestrel, EP-1 (quinestrol:levonorgestrel = 3:6), and control) were randomly assigned to the 16 sample plots. Control ware conducted 
at every April. Fitting with their experimental data, they found that $a=70.4, b=71.7$, $c=58.3$, and $d=17.1$. They pointed out the contraception rate with quinestrol is about 0.78 . From experience, $\varepsilon=0.24$.

The symmetrical effect of the contraception rate $p$ and the removal rate $q$ on determining whether the plateau pika population dies out is reflected in Figure 1. However, the effects of $p$ and $q$ on the behavior of approaching equilibrium are not symmetrical (see Figure 2).

We know that if only one control is implemented with the same control rate then the contraception control has better effect than the lethal control. Figures 3 and 4 show the dynamics of the population subject to the contraception control (dashed line) or the lethal control (solid line) with the same control rate 0.8. In Figure 3, control is implemented every 2 years and in Figure 4 control is implemented every year. We can see that the population with contraception control declines successively while the population with lethal control changes abruptly.

Usually, when the controlled population declines to a reasonable size, the harm is considered to be eliminated and no management is taken. In practice, it is impossible to eradicate all plateau pika completely in a relatively large area. A very small part of remnant population still exists. After all managements were stopped, the remnant

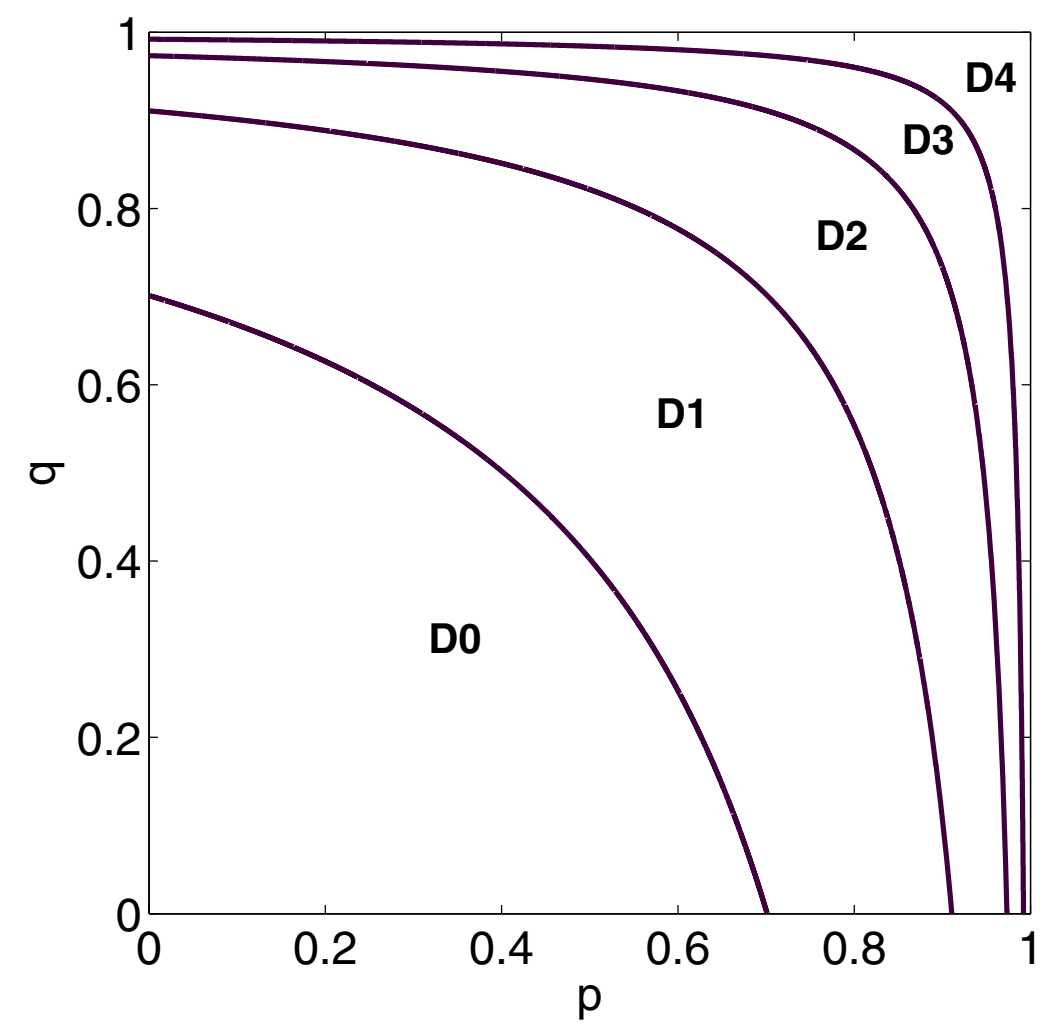

Figure 1 The symmetrical effect of $p$ and $q$ on determining whether the plateau pika population dies out. If $(p, q)$ locates in the domain $D 0$ then the population will persist and $E_{2}$ exists always, otherwise the population may die out. When $h=1$, if $(p, q)$ locates in D1UD2UD3UD4 then the population will die out. When $h=2$, if $(p, q)$ locates in D2 $\cup$ D3 $\cup$ D4 then the population will die out. Similar explanations can be given to $h=3$ and 4 . 


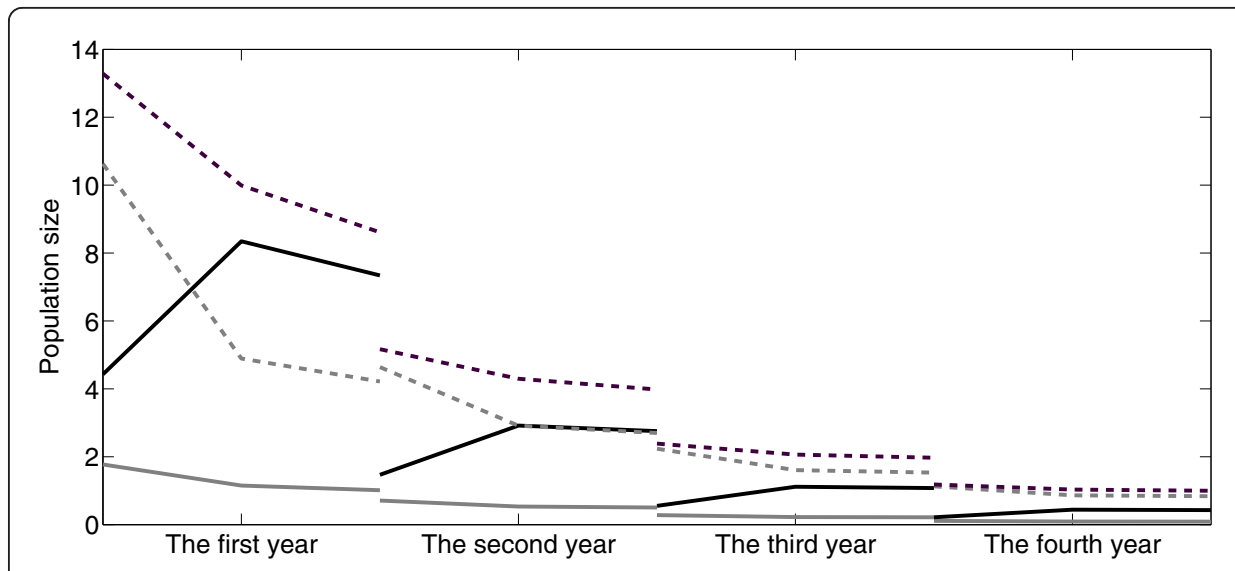

Figure 2 Non-symmetrical effects of $p$ and $q$ on the behavior of approaching equilibrium. The control was done at every April and the value of $\bar{f}_{n}$ and $\overline{\boldsymbol{s}}_{n}$ at the initial April are 22.14 and 0 ,

respectively. The black and the grey lines, respectively, represent the population size and the amount of the sterile individuals in the case where $p=0.4$ and $q=0.8$, while the black dashed line and the grey dashed line, respectively, stand for the population size and the amount of sterile individuals in the case where $p=0.8$ and $q=0.4$.

population would recover rapidly to high density (see Figure 5 ). Here, we take $\bar{f}_{0}=1$ and $\bar{s}_{0}=0.1$.

\section{Discussion}

In this article, discrete models of the plateau pika are formulated to account for the effects of the lethal and the contraception controls. In the models, we also

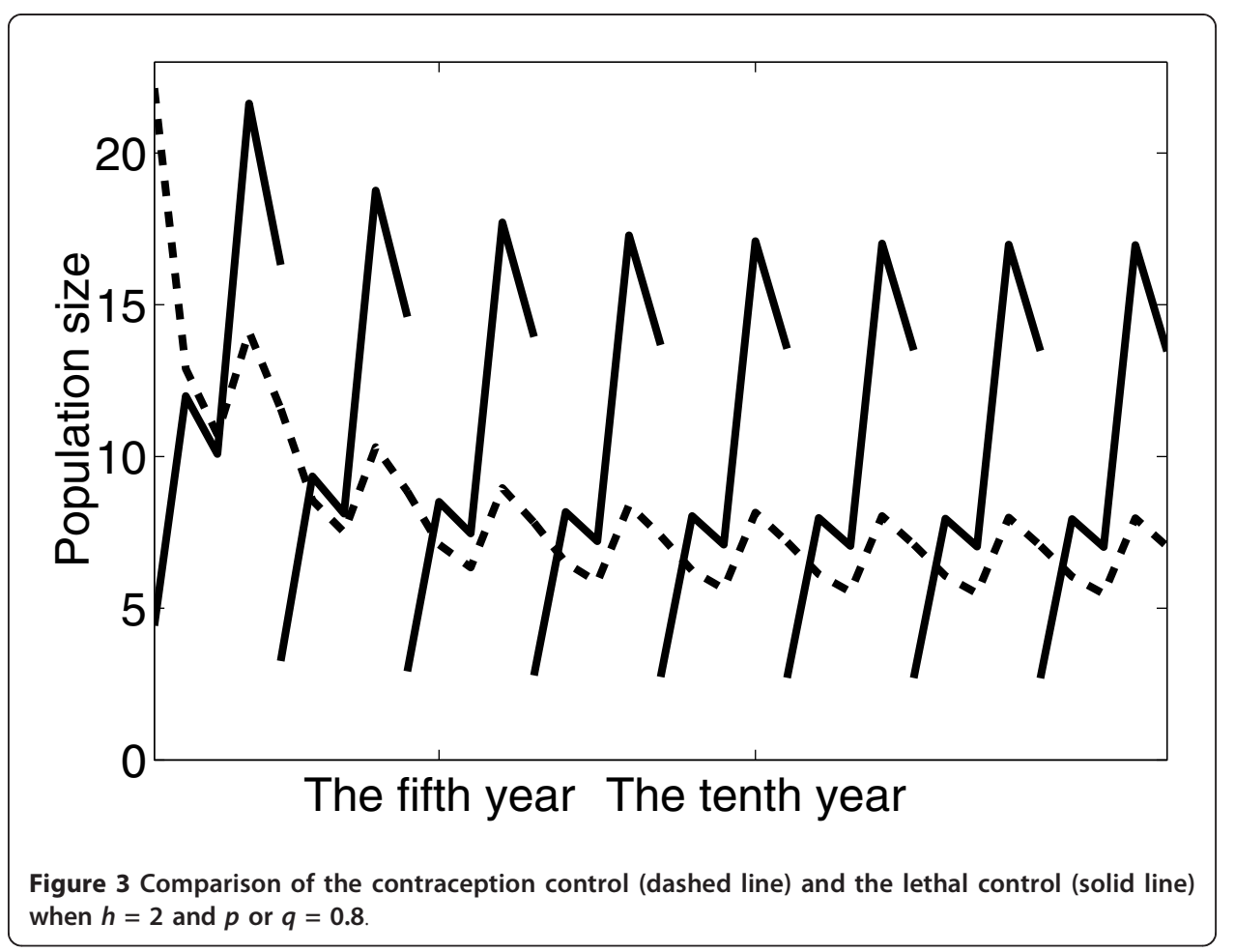




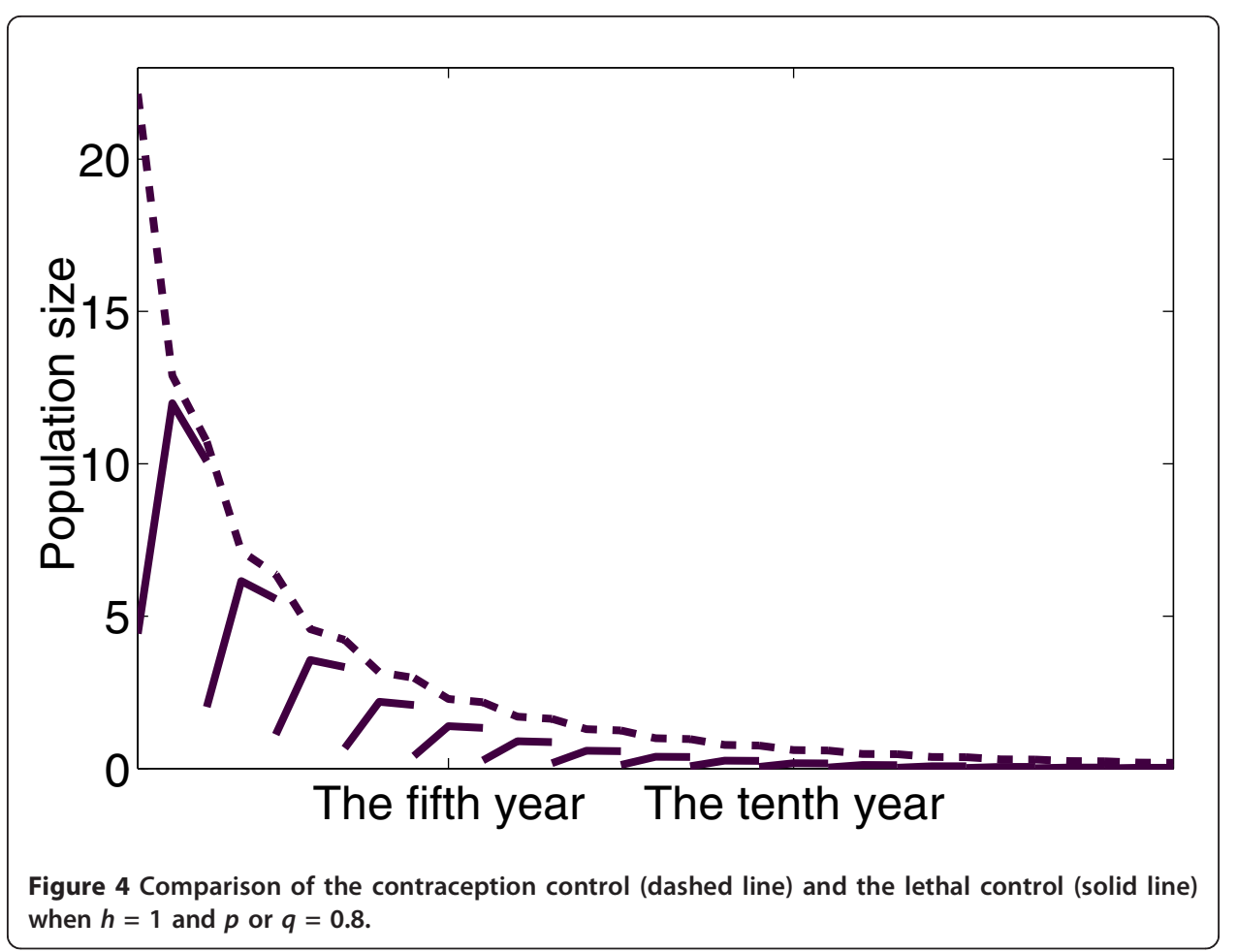

incorporated the seasonal cycle of breeding and non-breeding. The dynamics is completely studied and the impacts of the controls are analyzed.

For a better effect of control, we need larger contraception rate, or larger removal rate, or shorter control period. This makes the population small or even extinct.

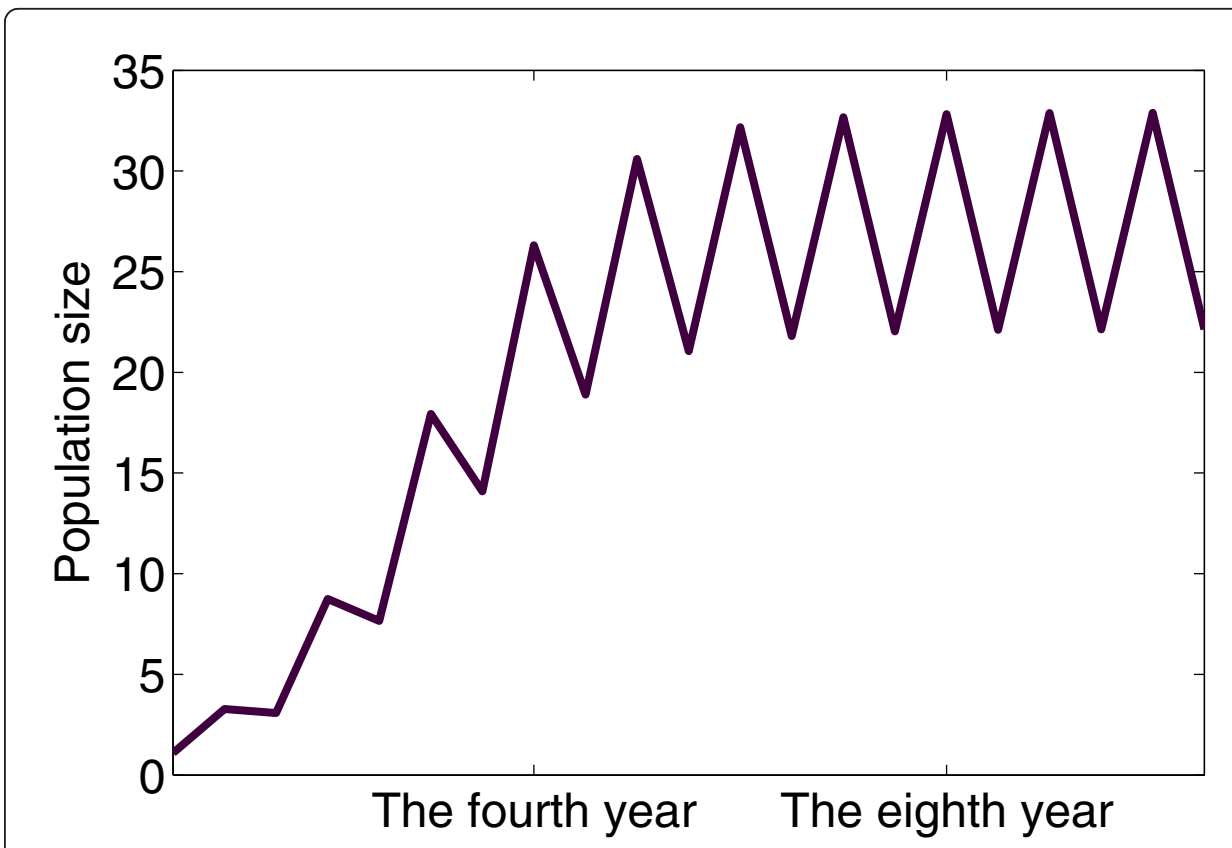

Figure 5 After all managements were stopped, the remnant population would recover rapidly where $\bar{f}_{0}=1$ and $\bar{s}_{0}=0.1$. 
Though the contraception rate and the removal rate have the same effect on determining whether the population dies out, their effects do not superimpose. Moreover, the two controls are combined, will give better result than only one control is implemented.

Among various methods to control rodent pests, the contraception and lethal controls possess distinct features. With contraception control, the population declines continuously, whereas with lethal control it reaches a small size after abrupt decreasing and increasing repeatedly. Plateau pika is an important component of the alpine meadow ecosystem. On the one hand, abrupt change of its population size would result in turbulence of material flow, energy flow, and information flow in the ecosystem. When the fragile ecosystem cannot endure this kind of disturbance it maybe disintegrate. So, it is preferable to let the plateau pika population reduce gradually. On the other hand, recovery of degraded alpine meadow is a lengthy process. Plateau pika, as the leading pest, accelerates the degradation of the alpine meadow. Therefore, it is urgent to let the plateau pika population reduce rapidly. This contradiction can be solved by implementing contraception control and lethal control simultaneously. Despite the better final effect of contraception control, the contraception and lethal controls cannot replace with each other. Combined implementation will give more reasonable result.

With certain control strategy, the plateau pika population will decline to a small size. Some occasional accidents may cause the small size population to die out. However, one should not be optimistic. Even if the plateau pika population died out, the left degraded alpine meadow is still a favorable habitat of plateau pika. In practice, when the plateau pika population is too small to cause damage, control will be stopped because of concerns about manpower, finance and material. The remaining and immigratory plateau pika will recover rapidly (see Figure 5). In this sense, contraception control or lethal control can only control the harm caused by overabundant plateau pika for a while but cannot root out the harm.

The degraded alpine meadow provides the plateau pika with a suitable habitat while the overabundant plateau pika population aggravates the degradation of the alpine meadow [2]. The long-term degradation of the alpine meadow will greatly reduce its ecological function, whereas the temporary reduction of plateau pika is helpless for the recovery of the degraded alpine meadow. Therefore, alternate methods like establishing artificial or semiartificial grassland are applied to manage the degraded alpine meadow [4]. After restoring the vegetation, the alpine meadow is no longer a suitable habitat for plateau pika, namely, the carrying capacity of plateau pika decreases. From the analysis in Sections 2 and 3, no matter whether there is control or not, the population size at the positive equilibrium reduces as long as the carrying capacity decreases. Therefore, restoring vegetation is the fundamental method to root out plateau pika.

The models formulated in this article bear strong practicability. All parameters can be estimated from data collected from field experiments. The population size at April and August can be calculated from (6). The population size at other months can be obtained through interpolating with an appropriate interpolation function like $a \exp (b t)$.

Besides its own law, the dynamics of plateau pika is also affected by climatic factors such as precipitation and temperature. Because of the randomness of climatic factors, the amount of plateau pika may experience big annual variation [16]. As a result, we 
should frequently monitor plateau pika. When the population size reaches a threshold, we should take control strategies in time.

\section{Acknowledgements}

This research was supported partially by the Natural Science Foundation of Shanxi Province (2009011005-3), by the National Natural Science Foundation of China (11071283), by the Yuncheng university research projects (YQ-2011046), by the Natural Sciences and Engineering Research Council of Canada (NSERC), by the Ontario Early Researcher Award Program, and by the One Hundred Talents Project of Shanxi Province.

\section{Author details}

${ }^{1}$ Electromechanical Engineering College, North University of China, Taiyuan 030051, China ${ }^{2}$ College of Science, North University of China, Taiyuan 030051, China ${ }^{3}$ Department of Applied Mathematics, Yuncheng University, Yuncheng 044000, China ${ }^{4}$ Department of Mathematics, Wilfrid Laurier University, Waterloo, ON N2L 3C5, Canada

\section{Authors' contributions}

ZJ directed the study and helped inspection. HL established the models and carried out the main results of this article. FZ performed the numerical simulation. YC drafted the manuscript. All the authors read and approved the final manuscript.

\section{Competing interests}

The authors declare that they have no competing interests.

\section{Received: 2 February 2012 Accepted: 8 March 2012 Published: 8 March 2012}

\section{References}

1. Lai, CH, Smith, AT: Keystone status of plateau pikas (Ochotona curzoniae): effect of control on biodiversity of native birds. Biodivers Conserv. 12(9):1901-1912 (2003). doi:10.1023/A:1024161409110

2. Liu, HW, Zhou, L: Modeling dispersal of the plateau pika (Ochotona curzoniae) using a cellular automata model. Ecol Model. 202(3-4):487-492 (2007). doi:10.1016/j.ecolmodel.2006.11.010

3. Pech, RP, Jiebu, AD, Zhang, Y, Lin, H: Population dynamics and responses to management of plateau pikas Ochotona curzoniae. J Appl Ecol. 44(3):615-624 (2007). doi:10.1111/j.1365-2664.2007.01287.x

4. Zhou, H, Zhao, X, Tang, Y, Gu, S, Zhou, L: Alpine grassland degradation and its control in the source region of the Yangtze and Yellow rivers China. Jpn Soc Grassland Sci. 51(3):191-203 (2005)

5. Singleton, GR, Hinds, LA, Leirs, H, Zhang, Z: Ecologically-Based Management of Rodent Pests. Arawang Communication Group, Canberra (1999)

6. Jing, Z, Wang, Q, Shi, H, Ma, Y, Shi, J: The poison effect experiment of botulin model D for plateau pika (Ochotona curzoniae). Pratacult Sci. 23(3):89-91 (2006)

7. Knipling, EF: Sterile male method of population control. Science. 130(3380):902-904 (1959). doi:10.1126/ science.130.3380.902

8. Knipling, EF: Use of insects for their own destruction. J Econ Entomol. 53(3):415-420 (1960)

9. Chen, Q, Qu, J, Liu, M, Zhang, Y: Palatability of anti-fertility baits containing compounds of quinestrol, levonorgestrel and EP-1 on Ochotona curzoniae. Chin J Zool. 45(3):87-90 (2010)

10. Wei, W, Fan, N, Zhou, W, Yang, S, Jing, Z, Cao, Y: The control effect of compound sterilant on population of plateau pika. Acta Agrestia Sinica. 7(1):39-45 (1999)

11. Zhang, Z: Mathematical models of wildlife management by contraception. Ecol Model. 132(1):105-113 (2000) doi:10.1016/S0304-3800(00)00308-2

12. Smith, AT, Ivins, BL: Reproductive tactics of pikas: why have two litters? Can J Zool. 61(7):1551-1559 (1983). doi:10.1139/ z83-209

13. Burkey, TV, Stenseth, NC: Population dynamics of territorial species in seasonal and patchy environments. Oikos. 69(1):47-53 (1994). doi:10.2307/3545282

14. Liu, H, Zhou, L, Liu, W, Zhou, H: Dynamics model for population whose growing and non-growing season present in turns and the influence of environmental change. J Biomath. 23(3):435-442 (2008)

15. Liu, M, Qu, J, Yang, M, Wang, Z, Wang, Y, Zhang, Y, Zhang, Z: Effects of quinestrol and levonorgestrel on populations of plateau pikas, Ochotona curzoniae, in the Qinghai-Tibetan Plateau. Pest Manag Sci (2012). doi:10.1002/ps.2302

16. Zhang, W, Ding, L, Han, T: Effect of precipitation on the variation of Ochotona curzoniae population. Pratacult Sci. 16(6):20-22 (1999)

doi:10.1186/1687-1847-2012-29

Cite this article as: Liu et al:: Population dynamics of plateau pika under lethal control and contraception control. Advances in Difference Equations 2012 2012:29. 